$\mathrm{Hb}$ binding to megalin/cubilin receptors. We have developed a highly sensitive fluorescence-based assay to test for such compounds. Inhibition of DBP uptake and vitamin D metabolism in the PT could lead or contribute to vitamin D deficiency. To our knowledge, our study is the first to suggest a mechanism for vitamin D deficiency commonly observed in SCD patients. Ongoing studies focus on measuring vitamin D metabolism in both cell and mouse models of SCD.

3236

Identification of exhaustive markers in cytotoxic T-cells to guide immune modulation in hepatocellular carcinoma ex vivo

Lauren Norell Krumeich ${ }^{1}$, Tatiana Akimova, Jason Stadanlick, Abhishek Rao, Neil Sullivan, Seth Concors, Paul Hernandez, David Aufhauser Jr, Evgeniy Eruslanov, Wayne Hancock and Matthew Levine

${ }^{1}$ University of Pennsylvania School of Medicine

OBJECTIVES/SPECIFIC AIMS: Objective: apply checkpoint inhibitors that are specific to the exhaustive markers expressed on tumor CD8+ T-cells ex vivo in order to improve cytokine release and cytotoxic function in comparison to two control groups: (1.) T-cells that receive no antibodies; (2.) T-cells that receive standard inhibition with PD-1 and CTLA-4 antibodies only. Long-term objective: provide personalized medicine in the treatment of HCC by using checkpoint inhibitors that are specific to the receptors expressed by an individual tumor. METHODS/STUDY POPULATION: The study population includes patients undergoing liver transplantation or surgical resection for HCC. Two grams of tumor, two grams of healthy liver tissue at least one centimeter from the tumor margin, and 50 milliliters of blood will be obtained. Solid tissue will be mechanically and enzymatically disrupted and CD8 + T-cells will be isolated from all sites. Using flow cytometry, the expression of surface receptors PD-1, CTLA-4, LAG-3, TIM-3, BTLA, CD244, and CD160 will be categorized in each tissue to identify which receptors are upregulated in the tumor microenvironment. Up to three antibodies specific to the upregulated receptor(s) on the tumor T-cells will be applied per specimen. The experimental arm will receive these antibodies and co-stimulation with CD3/CD28 and will be compared to two controls. One control will receive only $\mathrm{CD} 3 / \mathrm{CD} 28$, and the other will receive CD3/CD28 in addition to the standard combination of PD-1 and CTLA-4 inhibitors. From each condition, flow cytometry will be used to assess the mean production of interleukin-2, tumor necrosis factor$\alpha$, interferon $-\gamma$, granzyme $B$, and perforin expression as an assessment of T-cell function. RESULTS/ANTICIPATED RESULTS: Preliminary data from the peripheral blood of healthy controls confirms that the developed flow cytometry panels effectively identify the surface receptors and cytokine production of CD8+ T-cells. Two patients have successfully been enrolled in this study. It is predicted that T-cells extracted from the tumor will express more inhibitory receptors than normal liver or peripheral blood and will have increased function after they are targeted with checkpoint inhibitors that are specific to the inhibitory surface receptors they express. DISCUSSION/ SIGNIFICANCE OF IMPACT: HCC is the second leading cause of cancer-related death worldwide and therapeutic options are limited for patients who are not surgical candidates. T-cells are a critical component of the anti-tumor response to HCC. However, T-cells can develop an exhausted phenotype characterized by up-regulated inhibitory receptors (PD-1, CTLA-4, LAG-3, TIM-3, CD-244, CD-160, BTLA) and decreased function, allowing for immune escape. Clinical trials using combined checkpoint inhibition with PD-L1 and
CTLA- 4 antibodies have been considered a breakthrough for patients with advanced HCC, as up to $25 \%$ show an objective tumor response. The explanation for the varied susceptibility to checkpoint inhibition remains unknown and is hypothesized to be secondary to inconsistencies in the expression of surface inhibitory receptors. Although inhibitory receptor expression has been shown to be upregulated under conditions of hepatitis and/or HCC, there has been no single study to effectively investigate the expression of all known inhibitors in order to better explore the interplay between them. It will be of great academic interest and clinical purpose to evaluate individual receptor expression and engage the correlating antibodies given the possibility of synergism between receptors and the need for a more profound anti-tumor T-cell response in HCC.

3343

\section{Identification of host-microbial interaction networks that mediate intestinal epithelial barrier function in necrotizing enterocolitis}

David R Hill ${ }^{1}$, Roberto Cieza, Veda K. Yadagiri, Phillip Tarr,

Jason R. Spence and Vincent B. Young

${ }^{1}$ University of Michigan School of Medicine

OBJECTIVES/SPECIFIC AIMS: The central goal of this proposal is to characterize the mechanisms that mediate success or failure of immature intestinal barrier in necrotizing enterocilitis. METHODS/ STUDY POPULATION: To do this, I will utilize stem cell derived human intestinal organoids (HIOs), an innovative model of the immature intestine, and a cohort of bacterial isolates collected from premature infants who developed NEC to interrogate the cause-effect relationship of these strains on maintenance of the intestinal barrier. I hypothesize that the epithelial response to bacterial colonization is strain-dependent and results in differences in inflammatory signaling that shape epithelial barrier function in the immature intestine. RESULTS/ANTICIPATED RESULTS: Preliminary data shows that colonization of HIOs with different bacteria leads to species-specific changes in barrier function, and some species selectively damage the epithelial barrier while others enhance epithelial barrier function. I have identified key inflammatory signals that serve as central drivers of intestinal barrier function. DISCUSSION/SIGNIFICANCE OF IMPACT: Characterization of this process is expected to substantially advance scientific understanding of early events in NEC pathogenesis and lead to new opportunities for targeted therapeutic intervention to accelerate barrier maturation or prevent hyperinflammatory reactivity in the neonatal intestine. The research proposed in this application represents an entirely novel approach to studying host-microbial interactions in the immature. Conceptually, this novel translational approach will help to define the pivotal role of colonizing bacteria in initiating epithelial inflammation in NEC patients.

3253

Identification of Immune Cell Profiles and Molecular Pathways in Inflammatory Bowel Disease Driving Non-Response to Biologic Therapy

Harrison Michael Penrose ${ }^{1}$, Hani Nakhoul, Nathan Ungerleider,

Erik Flemington and Suzana Savkovic

${ }^{1}$ Tulane University School of Medicine- LA CaTS

OBJECTIVES/SPECIFIC AIMS: Inflammatory Bowel Disease (IBD) is a chronic, life-long condition characterized by inflammation of the intestine that greatly affects an individual's quality of life. While 
biologic therapy directed against $\mathrm{TNF} \alpha$ (anti-TNF $\alpha$, Infliximab) and $\alpha 4 \beta 7$ integrin (anti- $\alpha 4 \beta 7$; Vedolizumab) is used to treat IBD, a substantial number of patients remain non-responsive. Using a comprehensive bioinformatics approach, the aim of this study was to characterize immune cell profiles and altered molecular pathways in IBD patient non-responders to anti-TNF $\alpha$ and anti- $\alpha 4 \beta 7$ therapy to determine potential mechanisms and/or indicators of treatment nonresponse. METHODS/STUDY POPULATION: Publicly available whole transcriptomes from 65 healthy control and IBD endoscopic biopsies were assessed (NCBI GEO GSE73661). Specifically, transcript profiles from responders or non-responders to anti-TNF $\alpha$ and anti$\alpha 4 \beta 7$ therapy were utilized. Differentially expressed transcript profiles were obtained by comparing responders or non-responders prior to receiving therapy versus healthy controls using NCBI's GEO2R after adjustment with Benjamini and Hochberg testing $(\mathrm{p}<0.05)$. Immune profiling of DEgs were analyzed by the core LM22 immune signature for subsets of B-, T-, dendritic-, mast-cells, macrophages, and neutrophils (CIBERSORT, cibersort.stanford.edu) ( $\mathrm{p}<0.05)$. Networks, functional analysis, and interpretation of transcriptomic data were performed using Ingenuity Pathway Analysis (IPA) (Qiagen) $(\mathrm{p}<0.05)$. RESULTS/ANTICIPATED RESULTS: Initially, we determined colonic immune profiles in responders and non-responders to anti-TNF $\alpha$ and anti- $\alpha 4 \beta 7$ therapy. Compared to responders, in both anti-TNF $\alpha$ and anti- $\alpha 4 \beta 7$ non-responders we found elevated neutrophil levels $(\mathrm{p}<0.05)$. Specific to anti-TNF $\alpha$ treatment, non-responders demonstrated substantially reduced Treg cells $(\mathrm{p}<0.05)$; whereas, exclusive to anti- $\alpha 4 \beta 7$ treatment, non-responders showed elevated dendritic cells, activated CD4 T cells, and reduced M2 macrophages $(\mathrm{p}<0.05)$. Next we profiled differentially expressed transcripts to determine molecular pathways associated with therapy non-response. In both anti-TNF $\alpha$ and anti- $\alpha 4 \beta 7$ non-responders, we observed alterations in pathways specific to cellular growth and metabolism. Among cell growth pathways we found activated growth hormone, Wnt, ErB, and IGF-1 signaling; whereas, among metabolic regulation we found altered triglyceride, tryptophan, and leptin signaling. Moreover, unique to anti-TNF $\alpha$ non-responders, we found activated sphinogosine-1phosphate and paxillin pathways. While non-response to anti- $\alpha 4 \beta 7$ indicated activation of SAPK/JNK and IL-9 signaling. DISCUSSION/ SIGNIFICANCE OF IMPACT: Together these data define specific immune profiles and molecular pathways observed in non-responders to anti-TNF $\alpha$ and anti- $\alpha 4 \beta 7$ therapy. Our analysis identified substantial alterations in pathways specific to cellular growth and metabolism, identifying a link between non-response to biologic therapy and specific cell functions. These data suggest particular alterations in immune profiles and molecular pathways could play a role in non-response to biologic therapy, highlighting a future direction for personalized treatment regimens that could lead to more targeted use of existing therapies and more favorable patient health outcomes.

3005

Integrin Mac-1 Potentiates Neutrophil Adhesion and NET Release in Antiphospholipid Syndrome

Gautam Sule ${ }^{1}$, William J. Kelley ${ }^{3}$, Srilakshmi Yalavarthi², Omolola Eniola-Adefeso ${ }^{3}$ and Jason S. Knight ${ }^{1}$

${ }^{1}$ University of Michigan School of Medicine; ${ }^{2}$ Department of Internal Medicine, University of Michigan and ${ }^{3}$ Department of Chemical Engineering, University of Michigan

OBJECTIVES/SPECIFIC AIMS: While the role of antiphospholipid antibodies in activating endothelial cells has been extensively studied, the impact of these antibodies on the adhesive potential of leukocytes has received considerably less attention. Mac-1 is a heterodimeric beta- 2 integrin primarily expressed by myeloid-lineage cells. In its activated state, Mac-1 mediates cell-cell interactions by engaging a variety of surface molecules, including the endotheliumexpressed glycoprotein ICAM-1. Here, our goals were (1) to determine the extent to which APS neutrophils adhere to healthy, resting endothelial cells under physiologic flow conditions, and (2) to identify potential therapeutic targets by elucidating the molecules required for that adhesion. METHODS/STUDY POPULATION: Primary APS patients (meeting Sydney criteria) and non-autoimmune controls were matched for age and gender. Freshly isolated human umbilical vein endothelial cells (HUVECs) were utilized within five passages. Samples were introduced into a flow channel via a programmable syringe pump, and perfused across a resting HUVEC monolayer. After 15 minutes of perfusion, the chamber was flushed, and the remaining adherent cells were quantified. Flow cytometry was used to identify differentially-expressed molecules on the surface of APS neutrophils. Neutrophil extracellular trap (NET) release was assessed in static neutrophil-HUVEC cultures. RESULTS/ANTICIPATED RESULTS: Pre-treating control neutrophils with APS plasma resulted in increased adhesion as compared with control plasma $(>2.5$-fold for $\mathrm{n}=12$ plasma samples; $\mathrm{p}<0.05)$. This was true under both venous conditions (low shear) and conditions representative of the microvasculature (pulsatile flow and higher shear). Control neutrophils treated with APS plasma demonstrated upregulation of CD64, CEACAM-1, beta-2 glycoprotein I, and activated Mac-1 on the neutrophil surface, as well as shedding of L-selectin. Upregulation of activated Mac-1 and shedding of L-selectin were also triggered by IgG purified from APS plasma. For these changes to be meaningful clinically, we reasoned that they should be present on neutrophils in the peripheral blood of APS patients. Indeed, perfusion of anticoagulated blood through the flow chamber resulted in increased adhesion of patient neutrophils as compared with controls ( $>5$-fold for $n=18$ patients; $p<0.05$ ). Similarly, patient neutrophils demonstrated upregulation of CD64, CEACAM-1, beta-2 glycoprotein I, and activated Mac-1 on the neutrophil surface. A monoclonal antibody specific for activated Mac-1 reduced the adhesion of APS neutrophils to HUVECs in the flowchamber assay ( $>2$-fold reduction for $n=5$ patients; $p<0.05$ ). Importantly, the same monoclonal antibody reduced NET release in neutrophil-HUVEC co-cultures. DISCUSSION/SIGNIFICANCE OF IMPACT: APS neutrophils have an increased adhesive potential, which is dependent upon the activated form of Mac-1. This may lower the threshold for both neutrophil-endothelium engagement and NET release in patients, and thereby have implications for events such as venous thrombosis. Studies are underway to determine the extent to which Mac-1 is a viable therapeutic target in preclinical models of APS.

3114

Investigating the therapeutic potential of parthenolide in the treatment of hematopoietic neoplasms in dogs Lisa J Schlein, ACVP ${ }^{1}$, Aubree Peterson ${ }^{2}$, Barbara Rose ${ }^{2}$ and Douglas Thamm²

${ }^{1}$ University of Colorado at Denver and ${ }^{2}$ Colorado State University

OBJECTIVES/SPECIFIC AIMS: Determine PTL's mechanism(s) of action in a panel of canine hematopoietic cell lines; this will enable us to 1 ) verify that PTL is working as expected and 2) rationally select combination therapeutics. Characterize the in vitro sensitivity of canine hematopoietic cell lines to PTL in combination with other 